\title{
Scalar-spinor fields interaction in de Sitter ambient space formalism
}

\author{
Y. Ahmadi,, , * F. Jalilifard, ${ }^{1, \oplus}$ and M.V. Takook ${ }^{1,+}$ \\ ${ }^{1}$ Department of Physics, Razi University, Kermanshah, Iran
}

\begin{abstract}
In de Sitter ambient space formalism, the massless minimally coupled scalar field can be constructed from a massless conformally coupled scalar field and a constant five-vector $A^{\alpha}$. Also, a constant five-vector $B^{\alpha}$ appears in the interaction Lagrangian of massless minimally coupled scalar and spinor fields in this formalism. These constant five-vector fields can be fixed in the interaction case in the null curvature limit. Here we will calculate the $\mathcal{S}$ matrix elements of scalar-spinor fields interaction in the tree level approximation. Then the constant five-vectors $A^{\alpha}$ and $B^{\alpha}$, will be fixed by comparing the $\mathcal{S}$ matrix elements in the null curvature limits with the Minkowskian counterparts.
\end{abstract}

\section{INTRODUCTION}

The interaction Lagrangian in standard model is usually constructed from gauge theory, but the scalar-spinor field interaction is presented by the Yukawa potential. In quantum field theory (QFT) the Yukawa potential cannot be written from any group theoretical point of view. In the previous paper, the interaction Lagrangian of scalar-spinor fields is defined by a new transformation in de Sitter (dS) ambient space formalism, which was free of any infrared divergence [1]. The Yukawa potential can be obtained from this Lagrangian in the null curvature limit. In this model two constant five-vector fields appear, one is from the new transformation and the other is from the massless minimally coupled $(m m c)$ scalar field two-point function [1]. In this article we will fixed this two constant five-vector fields by calculating the scattering $(\mathcal{S})$ matrix elements of the scalar-spinor fields interaction in the null curvature limit.

The experimental data confirm that the our universe can be described by the dS metric in the large scale and also in the inflationary epoch [2, 3]. Thus it is important to construct QFT in dS space-time. In last years, the efforts have been made to do it in ambient space formalism [4-10]. The advantage of using ambient space is due to the linearity of the group action on the ambient space formalism, then the calculations are very similar to the Minkowski counterpart.

We know that, in the Minkowski space-time, the analyticity properties of the two-point functions are the fundamental basis for calculation of the probability amplitude or the Green functions of the interaction fields. The analyticity properties of the two-point functions in dS ambient space formalism have been proved by Bros et al. [11 13]. Therefore, this formalism is more appropriate for constructed the quantum interaction fields in dS space-time. In the previous article, we studied the Compton scattering in $\mathrm{dS}$ ambient space formalism and showed that the $\mathcal{S}$ matrix elements in the null curvature limit reduce to the Minkowskian counterpart [14]. The interaction fields are important in this article for two reason: First, the effects of the classical gravitational field on the quantum field are better seen in the QFT in the dS ambient space formalism. Secondly, in the formulation of the QFT in dS ambient space formalism, two constant five-vector fields appear, which can be fixed in the null curvature limit $[1,4,10]$.

\footnotetext{
*Electronic address: ahmadi.pave@gmail.com

${ }^{\dagger}$ Electronic address: jalilifard@iau-ea.ac.ir

‡Electronic address: takook@razi.ac.ir
} 
The organization of this article is as follow. We present the notation and terminology that has been used in this article in section III. In section III, the interaction Lagrangian in the small curvature approximation has been presented and then the $\mathcal{S}$ matrix elements for the spinor-scalar interaction have been calculated in dS ambient space formalism. In section IV the null curvature limit of the $\mathcal{S}$ matrix element has been calculated and then the constant five-vectors $A^{\alpha}$ and $B^{\alpha}$ have been fixed in this limit. Finally, the conclusion has been presented in section $\mathrm{V}$,

\section{NOTATION AND TERMINOLOGY}

The dS space-time can be considered as a 4-dimensional hyperboloid embedded in 5-dimensional Minkowski space with the following relation:

$$
M_{H}=\left\{x \in \mathbb{R}^{5} \mid x \cdot x=\eta_{\alpha \beta} x^{\alpha} x^{\beta}=-H^{-2}\right\} ; \quad \alpha, \beta=0,1,2,3,4 .
$$

The dS metric is:

$$
d s^{2}=\left.\eta_{\alpha \beta} d x^{\alpha} d x^{\beta}\right|_{x^{2}=-H^{-2}}=g_{\mu \nu}^{d S} d X^{\mu} d X^{\nu} ; \mu=0,1,2,3,
$$

where $\eta_{\alpha \beta}=\operatorname{diag}(1,-1,-1,-1,-1), H$ is Hubble parameter, $X^{\mu}$ is dS intrinsic coordinate and $x^{\alpha}$ is the 5 -dimensional $\mathrm{dS}$ ambient space formalism $\left(x \cdot x=-H^{-2}\right)$. The ambient space coordinate $x^{\alpha}$ can be represented in terms of the intrinsic coordinate $X^{\mu}$. For defining time evolution operator and scattering matrix, one can choose the following static coordinate system:

$$
\left\{\begin{array}{l}
x^{0}=\sqrt{H^{-2}-r^{2}} \sinh H t_{s} \\
x^{1}=\sqrt{H^{-2}-r^{2}} \cosh H t_{s} \\
x^{2}=r \cos \theta \\
x^{3}=r \sin \theta \cos \phi \\
x^{4}=r \sin \theta \sin \phi
\end{array}\right.
$$

where $-\infty<t_{s}<\infty, 0 \leq r<H^{-1}, 0 \leq \theta \leq \pi, 0 \leq \phi<2 \pi$. This coordinate system does not covered the total of the $\mathrm{dS}$ hyperboloid. For investigating the null curvature limit, the following coordinate system is useful:

$$
\left\{\begin{aligned}
x^{0} & =H^{-1} \sinh \left(H X^{0}\right), \\
\vec{x} & =H^{-1} \frac{\vec{X}}{\|\vec{X}\|} \cosh \left(H X^{0}\right) \sinh (H\|\vec{X}\|), \\
x^{4} & =H^{-1} \cosh \left(H X^{0}\right) \cosh (H\|\vec{X}\|),
\end{aligned}\right.
$$

where the $\|\vec{X}\|=\left(X_{1}^{2}+X_{2}^{2}+X_{3}^{3}\right)^{\frac{1}{2}}$ is the norm of three-vector $\vec{X}$.

The Fourier transformation cannot be generally defined in curved space-time, but in the dS spacetime, due to the maximally symmetric properties of dS hyperboloid the Fourier-Helgason-type transformation or the Bros-Fourier transformation can be defined [12, 13, 15, 16]. Then, corresponding to any space-time variable $x^{\alpha}$ another transformed variable, $\xi^{\alpha}=\left(\xi^{0}, \vec{\xi}, \xi^{4}\right)$ can be defined in the positive null-cone $C^{+}=\left\{\xi \in \mathbb{R}^{5} \mid \xi \cdot \xi=0, \quad \xi^{0}>0\right\}$ [12, 13]. One can parameterize the $\xi$ in terms of massive particle four-momentum in flat Minkowski space-time as:

$$
\xi=\left(\xi^{0}, \vec{\xi}, \xi^{4}\right)=\left(\frac{k^{0}}{m}=\sqrt{\frac{\vec{k}^{2}}{m^{2}}+1}, \frac{\vec{k}}{m},-1\right) .
$$


In the dS ambient space formalism, dS-Dirac first-order field equation is [17]:

$$
\left(-i \not x \not \partial^{\top}+2 i \pm \nu\right) \psi(x)=0,
$$

where $\not x=\eta_{\alpha \beta} \gamma^{\alpha} x^{\beta}$ and $\partial_{\alpha}^{\top}=\theta_{\alpha \beta} \partial^{\beta}=\partial_{\alpha}+H x_{\alpha} x \cdot \partial . \theta_{\alpha \beta}=\eta_{\alpha \beta}+H^{2} x_{\alpha} x_{\beta}$ is the projection operator on dS hyperboloid. In this equation, $\nu$ is related to dS mass parameter $m_{f, \nu}^{2}=H^{2}\left(2+\nu^{2} \pm i \nu\right)$ and in the null curvature limit it is defined the Minkowskian mass parameter $m$ [10]:

$$
\lim _{H \rightarrow 0, \nu \rightarrow \infty}(H \nu)^{2}=m^{2}
$$

The five matrices $\gamma^{\alpha}$, which satisfy the conditions $\gamma^{\alpha} \gamma^{\beta}+\gamma^{\beta} \gamma^{\alpha}=2 \eta^{\alpha \beta}$ and $\gamma^{\alpha \dagger}=\gamma^{0} \gamma^{\alpha} \gamma^{0}$, can be chosen as [4, [8, 17]:

$$
\begin{gathered}
\gamma^{0}=\left(\begin{array}{cc}
I & 0 \\
0 & -I
\end{array}\right), \quad \gamma^{4}=\left(\begin{array}{cc}
0 & I \\
-I & 0
\end{array}\right), \\
\gamma^{1}=\left(\begin{array}{cc}
0 & i \sigma^{1} \\
i \sigma^{1} & 0
\end{array}\right), \gamma^{2}=\left(\begin{array}{cc}
0 & -i \sigma^{2} \\
-i \sigma^{2} & 0
\end{array}\right), \gamma^{3}=\left(\begin{array}{cc}
0 & i \sigma^{3} \\
i \sigma^{3} & 0
\end{array}\right),
\end{gathered}
$$

where $\sigma^{i}(i=1,2,3)$ are the Pauli matrices. These matrices are different from Minkowski gamma matrices $\gamma^{\prime \mu}$. The relation between the $\gamma$ matrices in Minkowski and dS ambient space formalism are [17]:

$$
\gamma^{\prime \mu}=\gamma^{\mu} \gamma^{4}
$$

The charged spinor field operator, which satisfies the equation (II.4), is [10]:

$$
\psi(x)=\int_{S^{3}} d \mu(\xi) \sum_{n}\left[a(\tilde{\xi}, n)(H x . \xi)^{-2-i \nu} \mathcal{U}(x, \xi, n)+b^{\dagger}(\xi, n)(H x . \xi)^{-1+i \nu} \mathcal{V}(x, \xi, n)\right]
$$

where $n=-\frac{1}{2}, \frac{1}{2}, \mathrm{~d} \mu(\xi)$ is the $S O(4)$-invariant normalized volume element and $\tilde{\xi}^{\alpha}=\left(\xi^{0},-\vec{\xi}, \xi^{4}\right)$. The explicit form of $\mathcal{U}$ and $\mathcal{V}$ has been defined in [4, 17]. The adjoint spinor $\bar{\psi}(x)$ in ambient space formalism is defined as $\bar{\psi}(x)=\psi^{\dagger}(x) \gamma^{0} \gamma^{4}$ [17]. The $a(\tilde{\xi}, n), a^{\dagger}(\tilde{\xi}, n)$ and $b(\tilde{\xi}, n), b^{\dagger}(\tilde{\xi}, n)$ are annihilation and creation operators [10]:

$$
a^{\dagger}(\xi, n)|\Omega\rangle \equiv\left|1_{\xi, n}^{a}\right\rangle, \quad b^{\dagger}(\xi, n)|\Omega\rangle \equiv\left|1_{\xi, n}^{b}\right\rangle .
$$

The vacuum state $|\Omega\rangle$, with norm $\langle\Omega \mid \Omega\rangle=1$, is invariant under the action of the UIR of the dS group. This vacuum state can be identified with the Bunch-Davies or Hawking-Ellis vacuum state. The anti-commutation relations for creation and annihilation operators are [10, 17]:

$$
\begin{aligned}
& {\left[a\left(\tilde{\xi}^{\prime}, n^{\prime}\right), a^{\dagger}(\xi, n)\right]_{+}=\mathcal{N}_{p}(\xi, n) \delta_{s^{3}}\left(\xi-\xi^{\prime}\right) \delta_{n n^{\prime}},} \\
& {\left[b\left(\tilde{\xi}^{\prime}, n^{\prime}\right), b^{\dagger}(\xi, n)\right]_{+}=\mathcal{N}_{q}(\xi, n) \delta_{s^{3}}\left(\xi-\xi^{\prime}\right) \delta_{n n^{\prime}},}
\end{aligned}
$$

where $\mathcal{N}_{p}(\xi, r) \equiv \mathcal{N}_{p}$ and $\mathcal{N}_{q}(\xi, r) \equiv \mathcal{N}_{q}$ are the normalization constants. 
In dS ambient space formalism, the field equation for massless conformally coupled $(m c c)$ scalar field is [4, 10, 13]:

$$
\left(Q_{0}-2\right) \phi_{m c c}(x)=0,
$$

that $Q_{0}=-H^{-2} \partial^{\top} \cdot \partial^{\top} \equiv-H^{-2} \square_{H}$. The $m c c$ scalar field operator, which satisfies the field equation (II.10), is [10, 13]:

$$
\phi_{m c c}(x)=\sqrt{c_{0}} \int_{S^{3}} d \mu(\xi)\left[d(\tilde{\xi}, 1)(H x \cdot \xi)^{-2}+d^{\dagger}(\xi, 0)(H x \cdot \xi)^{-1}\right],
$$

where $c_{0}$ is the normalization constant. The $d(\tilde{\xi}, 1)$ and $d^{\dagger}(\xi, 0)$ are annihilation and creation operators respectively:

$$
d^{\dagger}(\xi, 0)|\Omega\rangle \equiv\left|1_{\xi}^{d}\right\rangle, \quad d(\tilde{\xi}, 1)|\Omega\rangle=0
$$

Between the massless minimally coupled $(m m c)$ scalar field, and the $m c c$ scalar fields in the dS ambient space formalism, the following relation exist [1, 10, 18, 19]:

$$
\phi_{m m c}(x)=\left[A \cdot \partial^{\top}+2 A \cdot H^{2} x\right] \phi_{m c c}(x),
$$

where $A^{\alpha}$ is an arbitrary constant five-vector. The $m m c$ scalar field operator can be obtained by inserting (II.11) in (II.12) and doing some simple calculations [1, 10]. The analytic field operator is defined in complex dS space-time $\phi_{m m c}(x)=\lim _{y \rightarrow 0} \phi_{m m c}(z)=\lim _{y \rightarrow 0} \phi_{m m c}(x+i y)$.

The analytic two-point function of $m c c$ scalar field can be calculated in terms of the generalized Legendre function [11, 13]:

$$
\begin{gathered}
W_{m c c}\left(z, z^{\prime}\right)=\left\langle\Omega\left|\phi_{m c c}(z) \phi_{m c c}\left(z^{\prime}\right)\right| \Omega\right\rangle= \\
c_{0} \int d \mu(\xi)(H z \cdot \xi)^{-2}\left(H z^{\prime} \cdot \xi\right)^{-1}=\frac{-H^{2}}{8 \pi^{2}} \frac{1}{1-\mathcal{Z}\left(z, z^{\prime}\right)}=\frac{1}{(2 \pi)^{2}} \frac{1}{\left(z-z^{\prime}\right)^{2}},
\end{gathered}
$$

where $\mathcal{Z}\left(z, z^{\prime}\right)=1+\frac{H^{2}}{2}\left(z-z^{\prime}\right)^{2}$ is the geodesic distance between two point $z$ and $z^{\prime}[13,20]$.

One can define the $m m c$ scalar analytic two-point function in terms of $m c c$ scalar analytic two-point function as [1, 10]:

$$
W_{m m c}\left(z, z^{\prime}\right)=\left[A \cdot \partial^{\top}+2 A \cdot H^{2} z\right]\left[A \cdot \partial^{\prime \top}+2 A \cdot H^{2} z^{\prime}\right] W_{m c c}\left(z, z^{\prime}\right) .
$$

For defining the real two-point function one can use the boundary value of the analytic two-point function $W_{m m c}\left(z, z^{\prime}\right)$ [10, 19],

$$
\mathcal{W}_{m m c}\left(x, x^{\prime}\right)=\lim _{y, y^{\prime} \rightarrow 0} W_{m m c}\left(z, z^{\prime}\right)=\lim _{y, y^{\prime} \rightarrow 0} W_{m m c}\left(x+i y, x^{\prime}+i y^{\prime}\right) .
$$

\section{SCATTERING MATRIX}

In ambient space formalism, the scalar field equation in dS space-time can be written as:

$$
\left(Q_{0}+\sigma(\sigma+3)\right) \phi(x)=0 .
$$


The solutions of this equation are named dS plane waves $(x \cdot \xi)^{\sigma}$. The five-vector $\xi^{\alpha}$ plays the role of momentum parameter and it is lying in the null-cone in ambient space [1]. The action of $m c c$ scalar and spinor free fields in dS universe is [1, 10, 17]:

$$
S(\psi, \phi)=\int \mathrm{d} \mu(x) \mathcal{L}(\psi, \phi)=\int \mathrm{d} \mu(x)\left[H \bar{\psi} \gamma^{4}\left(-i \not x \not \partial^{\top}+2 i\right) \psi+\phi Q_{0} \phi\right],
$$

where $\mathrm{d} \mu(x)$ is $\mathrm{dS}$ invariant volume element. The above action is invariant under the global $\mathrm{U}(1)$ symmetry. By defining the covariant derivative $D_{\alpha} \psi=\left(\partial_{\alpha}^{\top}+i \mathcal{G} B_{\alpha}^{\top} \phi_{m m c}\right) \psi$, where $B^{\alpha} B_{\alpha}=0$ and $\mathcal{G}$ is the coupling constant that determines interaction intensity, the action (III.15) will be invariant under the following transformations [1]:

$$
\left\{\begin{array}{c}
\phi \rightarrow \phi^{\prime}=\phi+(x . B)^{-3} \\
\psi \rightarrow \psi^{\prime}=e^{\frac{i}{2} \mathcal{G}(x \cdot B)^{-2}} \psi
\end{array} .\right.
$$

So by replacing this new derivative $D_{\alpha}$ with transverse derivative $\partial_{\alpha}^{\top}$, the interaction Lagrangian is obtained as [1]:

$$
\mathcal{L}_{i n t}=\mathcal{G} H \bar{\psi} \gamma^{4} \not x B^{\top} \phi_{m m c} \psi
$$

The constant five-vector $B^{\alpha}$ in Lagrangian and constant five-vector $A^{\alpha}$ in $m m c$ scalar two-point function can be fixed in the interaction case in the null curvature limit. The interaction Lagrangian plays an important role for calculation of the probability amplitude or the $\mathcal{S}$ matrix elements.

The time evolution operator cannot be explicitly defined in a general curved space time. In previous article we show that the time evolution operator $U\left(t, t_{0}\right)$, such that $|\alpha, t\rangle=U\left(t, t_{0}\right)\left|\alpha, t_{0}\right\rangle$, can be defined in the dS static coordinate system (II.1), but it is very complicated for calculation of the $\mathcal{S}$ matrix element [21]:

$$
\left|\phi_{\text {out }},+\infty>=U(\infty,-\infty)\right| \phi_{\text {in }},-\infty>=\mathcal{S} \mid \phi_{\text {in }},-\infty>
$$

Then we presented a logical approximation when the interaction occurs in the atomic dimension [14], which the $\mathcal{S}$ matrix elements can be calculated. In this approximation the direct effect of curvature can be ignored but the indirect effect is presented. In this case the time evolution operator can be expanded on the Minkowski space-time as [14]:

$$
U\left(t, t_{0}\right)=U_{M}\left(t, t_{0}\right)+H f\left(t, t_{0}\right)+\cdots,
$$

the term $f\left(t, t_{0}\right)$ is due to the direct effect of curvature that in atomic dimension and in null curvature limit, it is disappeared. The mathematical form of $U_{M}$ is exactly similar to the time evolution operator in Minkowskian space-time. Therefore the scattering matrix may be presented with the following approximate equation from the Minkowskian counterpart [22 24]:

$$
\mathcal{S}=\frac{(-1)^{\tau}}{\tau !} \sum_{\tau=0}^{\infty} \mathcal{S}^{(\tau)}+\cdots
$$

where

$$
\mathcal{S}^{(\tau)}=\int \mathrm{d} \mu\left(x_{1}\right) \int \mathrm{d} \mu\left(x_{2}\right) \cdots \int \mathrm{d} \mu\left(x_{\tau}\right) \quad T\left[\mathcal{L}_{i n t}\left(\phi\left(x_{1}\right)\right) \mathcal{L}_{i n t}\left(\phi\left(x_{2}\right)\right) \cdots \mathcal{L}_{i n t}\left(\phi\left(x_{\tau}\right)\right)\right] .
$$

In this equation, $\mathcal{L}_{\text {int }}(\phi(x))$ is the interaction Lagrangian and $T\left[\mathcal{L}_{\text {int }}\left(\phi\left(x_{1}\right)\right) \mathcal{L}_{\text {int }}\left(\phi\left(x_{2}\right)\right) \mathcal{L}_{\text {int }}\left(\phi\left(x_{3}\right)\right) \ldots\right]$ is the time order product of fields. 
By inserting interaction Lagrangian of spinor-scalar fields (III.16) in (III.18), the $\mathcal{S}^{(2)}$ is obtained as:

$$
\mathcal{S}^{(2)}=\mathcal{G}^{2} H^{2} \iint \mathrm{d} \mu(x) \mathrm{d} \mu\left(x^{\prime}\right) T\left[\left(\bar{\psi} \gamma^{4} \not x B^{\top} \phi_{m m c} \psi\right)_{x}\left(\bar{\psi} \gamma^{4} \not \not^{\prime} \not B^{\top} \phi_{m m c} \psi\right)_{x^{\prime}}\right] .
$$

By using Wick's theorem, one can write the time order product of fields in terms of the normal order product of fields. After some simple calculations, the time order product of (III.19) turns into 8 normal order product. Since we consider the interaction, which is depicted in figure 1, only one term is remained :

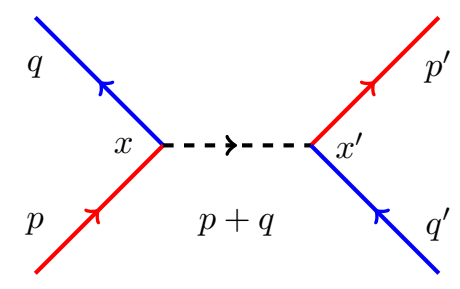

Figure 1: spinor-spinor interaction diagram with $m m c$ scalar propagator

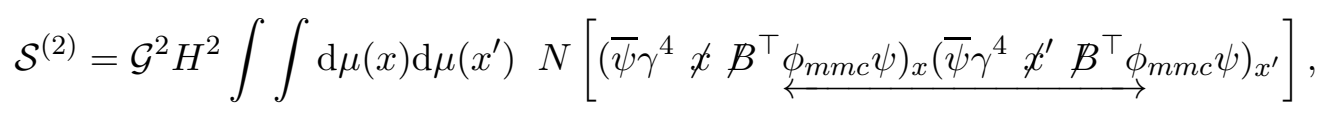

where, the symbol $\leftrightarrow$ is the $m m c$ scalar two-point function (II.14) and $N[. .$.$] is normal order product,$

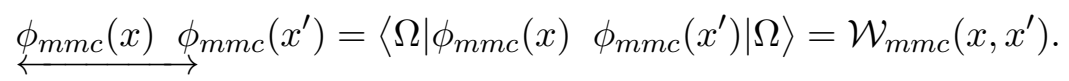

Since in (III.20) the spinor field, $\gamma$ matrices and other quantities are matrix, one can write them in terms of their components:

$$
\begin{aligned}
& \mathcal{S}^{(2)}=\mathcal{G}^{2} H^{2} \iint \mathrm{d} \mu(x) \mathrm{d} \mu\left(x^{\prime}\right) \mathcal{W}_{m m c}\left(x, x^{\prime}\right)\left(\gamma^{4} \not \not B^{\top}(x)\right)_{g h}\left(\gamma^{4} \not \not^{\prime} \not B^{\top}\left(x^{\prime}\right)\right)_{k l} \\
& \times N\left[\bar{\psi}_{g}(x) \psi_{h}(x) \bar{\psi}_{k}\left(x^{\prime}\right) \psi_{l}\left(x^{\prime}\right)\right] .
\end{aligned}
$$

By breaking the spinor field (II.7) into,+- parts, the normal order product in above relation (III.21) converted to 16 terms but just one of them is described the figure 1,

$$
\psi(x)=\psi^{+}(x)+\psi^{-}(x), \quad \bar{\psi}(x)=\bar{\psi}^{+}(x)+\bar{\psi}^{-}(x)
$$

physical term in normal order product $=\bar{\psi}_{g}^{-}(x) \psi_{h}^{+}(x) \psi_{l}^{-}\left(x^{\prime}\right) \bar{\psi}_{k}^{+}\left(x^{\prime}\right)$.

Thus the $\mathcal{S}^{(2)}$ is obtained as:

$$
\begin{aligned}
& \mathcal{S}^{(2)}=\mathcal{G}^{2} H^{2} \iint \mathrm{d} \mu(x) \mathrm{d} \mu\left(x^{\prime}\right) \mathcal{W}_{m m c}\left(x, x^{\prime}\right)\left(\gamma^{4} \not x B^{\top}(x)\right)_{g h}\left(\gamma^{4} \not x^{\prime} B^{\top}\left(x^{\prime}\right)\right)_{k l} \\
& \times\left\{\bar{\psi}_{g}^{-}(x) \psi_{h}^{+}(x) \psi_{l}^{-}\left(x^{\prime}\right) \bar{\psi}_{k}^{+}\left(x^{\prime}\right)\right\}
\end{aligned}
$$


In this interaction, incoming sate $\mid i>$ is two spinor fields with $\xi_{p}, \xi_{q}$ "momentums" and $r, n$ spin polarizations. Also outgoing state $\mid f>$ is two spinor fields with $\xi_{p}^{\prime}, \xi_{q}^{\prime}$ "momentums" and $r^{\prime}, n^{\prime}$ spin polarizations,

$$
\begin{gathered}
|i>=| 1_{p}^{a^{\dagger}}, 1_{q}^{b^{\dagger}}>=a_{r}^{\dagger}\left(\xi_{p}\right) b_{n}^{\dagger}\left(\xi_{q}\right) \mid \Omega> \\
|f>=| 1_{p^{\prime}}^{a^{\dagger}}, 1_{q^{\prime}}^{b^{\dagger}}>=a_{r^{\prime}}^{\dagger}\left(\xi_{p}^{\prime}\right) b_{n^{\prime}}^{\dagger}\left(\xi_{q}^{\prime}\right)|\Omega>\Longrightarrow<f|=<1_{p^{\prime}}^{a}, 1_{q^{\prime}}^{b}|=<\Omega| b_{n^{\prime}}\left(\xi_{q}^{\prime}\right) a_{r^{\prime}}\left(\xi_{p}^{\prime}\right) .
\end{gathered}
$$

Then the $\mathcal{S}_{f i}^{(2)}$ is:

$$
\begin{gathered}
\mathcal{S}_{f i}^{(2)}=<f\left|\mathcal{S}^{(2)}\right| i>=\mathcal{N}_{p} \mathcal{N}_{p^{\prime}} \mathcal{N}_{q} \mathcal{N}_{q^{\prime}} \mathcal{G}^{2} H^{2} \\
\times \iint \mathrm{d} \mu(x) \mathrm{d} \mu\left(x^{\prime}\right) \mathcal{W}_{m m c}\left(x, x^{\prime}\right)\left(\gamma^{4} \not x B^{\top}(x)\right)_{g h}\left(\gamma^{4} \not x^{\prime} B^{\top}\left(x^{\prime}\right)\right)_{k l} \\
\times\left(H x . \xi_{q}\right)^{-1-i \nu}\left(H x \cdot \xi_{p}\right)^{-2-i \nu}\left(H x^{\prime} \cdot \xi_{q}^{\prime}\right)^{-1+i \nu}\left(H x^{\prime} \cdot \xi_{p}^{\prime}\right)^{-2+i \nu} \\
\times \overline{\mathcal{V}}_{g}\left(x, \xi_{q}, n\right) \mathcal{U}_{h}\left(x, \xi_{p}, r\right) \overline{\mathcal{U}}_{k}\left(x^{\prime}, \xi_{p}^{\prime}, r^{\prime}\right) \mathcal{V}_{l}\left(x^{\prime}, \xi_{q}^{\prime}, n^{\prime}\right) .
\end{gathered}
$$

This $\mathcal{S}$ matrix elements may be calculated by numerical methods, but in this paper we would like to fix the constant five-vectors $A^{\alpha}$ and $B^{\alpha}$, which will be discussed in the next section.

\section{FLAT LIMIT}

The radius of dS hyperboloid is $H^{-1}$. The curvature of space-time diminishes for large radius, or equivalently $H \rightarrow 0$, and dS space-time matches with Minkowski space-time. In this limit one can obtain these relations [4]:

$$
\begin{aligned}
& \lim _{H \rightarrow 0}(x \cdot \xi)^{-2-i \nu}=e^{-i k \cdot X}, \quad \lim _{H \rightarrow 0} H \not x=\lim _{H \rightarrow 0} H \eta_{\alpha \beta} \gamma^{\alpha} x^{\beta}=-\gamma^{4}, \\
& \lim _{H \rightarrow 0} \theta_{\alpha \beta}=\lim _{H \rightarrow 0}\left(\eta_{\alpha \beta}+H^{2} x_{\alpha} x_{\beta}\right)=\eta_{\mu \nu}, \quad\left\{\begin{array}{c}
\mu=\nu=0,1,2,3 \\
\alpha=\beta=0,1,2,3,4 .
\end{array}\right.
\end{aligned}
$$

Since the five-vector $B^{\alpha}$ is constant, at null curvature limit $B^{4}$ can be chosen as zero and then

$$
\lim _{H \rightarrow 0} B^{\top}=-B_{\mu} \gamma^{\prime} \gamma^{4} ; \quad \mu=0,1,2,3
$$

The Minkowski $\gamma^{\prime}$ matrices are related to ambient $\gamma$ matrices as (II.6). The analytic $m m c$ scalar two-point function (II.14) in null curvature limit, $H \rightarrow 0$, is:

$$
\begin{gathered}
\lim _{H \rightarrow 0} W_{m m c}\left(z, z^{\prime}\right)=\lim _{H \rightarrow 0}\left[A \cdot \partial^{\top}+2 A \cdot H^{2} z\right]\left[A \cdot \partial^{\prime \top}+2 A \cdot H^{2} z^{\prime}\right] W_{m c c}\left(z, z^{\prime}\right) \\
=(A \cdot \partial)\left(A \cdot \partial^{\prime}\right) \lim _{H \rightarrow 0} W_{m c c}\left(z, z^{\prime}\right)
\end{gathered}
$$


and by using the (II.13), the real $m m c$ scalar two-point function in null curvature limit, $H \rightarrow 0$, obtains as:

$$
\lim _{H \rightarrow 0} \mathcal{W}_{m m c}\left(x, x^{\prime}\right) \equiv w\left(X, X^{\prime}\right)=\frac{1}{(2 \pi)^{2}}(A . \partial)\left(A . \partial^{\prime}\right) \frac{1}{\left(X-X^{\prime}\right)^{2}}
$$

By using this result for $m m c$ scalar field, adjoint spinor definition in ambient space formalism (II.7) and delta function definition in Minkowski space-time, $\delta^{4}\left(p-p^{\prime}\right)=\int \frac{\mathrm{d}^{4} X}{(2 \pi)^{4}} e^{-i X .\left(p-p^{\prime}\right)}$, the the null curvature limit of $\mathcal{S}_{f i}^{(2)}$ obtains as:

$$
\begin{aligned}
& \lim _{H \rightarrow 0} \mathcal{S}_{f i}^{(2)}=\frac{1}{(2 \pi)^{2}} \mathcal{N}_{p} \mathcal{N}_{p^{\prime}} \mathcal{N}_{q} \mathcal{N}_{q^{\prime}} \mathcal{G}^{2}\left\{\iint \mathrm{d}^{4} X \mathrm{~d}^{4} X^{\prime} e^{-i X .(p+q)} e^{i X^{\prime} \cdot\left(p^{\prime}+q^{\prime}\right)} \quad(A . \partial)\left(A . \partial^{\prime}\right) \frac{1}{\left(X-X^{\prime}\right)^{2}}\right\} \\
& \times\left(\mathcal{V}^{\dagger}(q, n) \gamma^{\prime 0}\right)_{g} B_{\mu}\left(\gamma^{\prime \mu} \gamma^{4}\right)_{g h} \mathcal{U}_{h}(p, r) \quad\left(\mathcal{U}^{\dagger}\left(p^{\prime}, r^{\prime}\right) \gamma^{\prime 0}\right)_{k} B_{\nu}\left(\gamma^{\prime \nu} \gamma^{4}\right)_{k l} \mathcal{V}_{l}\left(q^{\prime}, n^{\prime}\right) .
\end{aligned}
$$

By notice that $\int d^{4} X \partial_{\mu}\left(e^{-i X .(p+q)} \frac{1}{\left(X-X^{\prime}\right)^{2}}\right)=0$, one can prove the following relation:

$$
\begin{gathered}
\int d^{4} X e^{-i X \cdot(p+q)} \partial_{\mu}\left(\frac{1}{\left(X-X^{\prime}\right)^{2}}\right)=-\int d^{4} X \frac{1}{\left(X-X^{\prime}\right)^{2}}\left(\partial_{\mu} e^{-i X \cdot(p+q)}\right) \\
=-i(p+q)_{\mu} \int d^{4} X \frac{e^{-i X \cdot(p+q)}}{\left(X-X^{\prime}\right)^{2}}
\end{gathered}
$$

then we obtain:

$$
\begin{gathered}
\iint \mathrm{d}^{4} X \mathrm{~d}^{4} X^{\prime} e^{-i X .(p+q)} e^{i X^{\prime} \cdot\left(p^{\prime}+q^{\prime}\right)} \quad(A . \partial)\left(A . \partial^{\prime}\right) \frac{1}{\left(X-X^{\prime}\right)^{2}}= \\
A .(p+q) A \cdot\left(p^{\prime}+q^{\prime}\right) \iint \mathrm{d}^{4} X \mathrm{~d}^{4} X^{\prime} e^{-i X \cdot(p+q)} e^{i X^{\prime} \cdot\left(p^{\prime}+q^{\prime}\right)} \frac{1}{\left(X-X^{\prime}\right)^{2}} .
\end{gathered}
$$

After some straightforward calculations one can arrive the following relation:

$$
\iint \mathrm{d}^{4} X \mathrm{~d}^{4} X^{\prime} e^{-i X \cdot(p+q)} e^{i X^{\prime} \cdot\left(p^{\prime}+q^{\prime}\right)} \frac{1}{\left(X-X^{\prime}\right)^{2}}=(2 \pi)^{8} \delta\left(p+q-p^{\prime}-q^{\prime}\right) \frac{1}{(p+q)^{2}+i \epsilon} .
$$

By inserting these results in (IV.24) one can obtain the null curvature limit of $\mathcal{S}^{(2)}$ matrix as follow:

$$
\begin{gathered}
\lim _{H \rightarrow 0} \mathcal{S}_{f i}^{(2)}=(2 \pi)^{6} \mathcal{N}_{p} \mathcal{N}_{p^{\prime}} \mathcal{N}_{q} \mathcal{N}_{q^{\prime}} \mathcal{G}^{2} \delta\left(p+q-p^{\prime}-q^{\prime}\right) \\
\times\left(\mathcal{V}^{\dagger}(q, n) \gamma^{\prime 0}\right)_{g} B_{\mu}\left(\gamma^{\prime \mu} \gamma^{4}\right)_{g h} \mathcal{U}_{h}(p, r) \frac{[A .(p+q)]^{2}}{(p+q)^{2}+i \epsilon}\left(\mathcal{U}^{\dagger}\left(p^{\prime}, r^{\prime}\right) \gamma^{\prime 0}\right)_{k} B_{\nu}\left(\gamma^{\prime \nu} \gamma^{4}\right)_{k l} \mathcal{V}_{l}\left(q^{\prime}, n^{\prime}\right) .
\end{gathered}
$$

By comparing this equation with the Minkowski result [22], the constant five-vectors $A_{\mu}$ and $B_{\mu}$ can be fixed as:

$$
A_{\mu}=a \frac{\left(p_{\mu}+q_{\mu}\right)}{(p+q) \cdot(p+q)}, \quad B_{\mu}=b \gamma_{\mu}^{\prime} \gamma^{4}
$$

where $a$ and $b$ can be written in therms of the normalization constants. 


\section{CONCLUSION}

A constant five-vector field $A^{\alpha}$ appears in dS $m m c$ scalar field quantization. Also another constant five-vector field $B^{\alpha}$ appears in definition of the interaction Lagrangian of dS spinor-scalar fields interaction. These constant five-vectors $A^{\alpha}$ and $B^{\alpha}$ can be fixed in calculation of the $\mathcal{S}$ matrix elements in tree diagrams approximation in the null curvature limit. The constant five-vector $B^{\alpha}$ come from the gauge theory and depended on $\gamma$ matrices and the constant five-vector $A^{\alpha}$ come from the propagator. It is interesting to note that the constant five-vector $A^{\alpha}$ depended on the laboratory energy scale i.e. the energy-momentum of the incoming fields, which is a logical result. These calculations may be open the door to measuring the gravitational effects on quantum fields which may be measurable in the laboratory scale of energy. The other diagrams and interactions may be investigated in the next works.

Acknowledgments: The authors wish to express their particular thanks to M. Amiri, M. Rastiveis, R. Raziani and S. Tehrani-Nasab for discussions.

[1] J.P. Gazeau, M.V.Takook, (2016), Higgs Field and the Massless Minimally Coupled Scalar Field in de Sitter Universe arXiv:1612.08024, submitted to Class. Quant. Grav..

[2] S. Perlmutter, et al., Astrophys. J. 517, 565 (1999), Measurement of $\Omega$ and $\Lambda$ from 42 high-redshift supernovae, arXiv:astro-ph/9812133.

[3] A.G. Riess, et al., Astron. J. 116, 1009 (1998), Observational evidence from supernovae for an accelerating universe and a cosmological constant, arXiv:9805201v1.

[4] M.V. Takook, Thèse de l'université Paris VI (1997), Théorie quantique des champs pour des systèmes élémentaires "massifs" et de "masse nulle" sur l'espace- temps de de Sitter.

[5] M.V. Takook, A. Azizi, E. Babaian, Eur. Phys. J. C 72, 20206 (2012), Covariant Quantization of Massive Spin- $\frac{3}{2}$ Fields in the de Sitter Space, arXiv:1206.1997.

[6] S. Behroozi, S. Rouhani, M.V. Takook and M.R. Tanhayi, Phys. Rev. D 74, 124014 (2006), Conformally invariant wave equations and massless fields in de Sitter spacetime, arXiv:gr-qc/0512105.

[7] M. Enayati, S. Rouhani, M.V. Takook, Int J Theor Phys 56, 1068 (2017), Quantum Linear Gravity in de Sitter Universe On Gupta-Bleuler vacuum state, arXiv:1208.5562v1.

[8] M.V. Takook, Proceedings of the Group 21, 15-20 july (1996), Goslar, Germany, Spin $\frac{1}{2}$ field theory in the de Sitter space-time, arXiv:gr-qc/0005077.

[9] M. Dehghani, S. Rouhani, M.V. Takook and M.R. Tanhayi, Phys. Rev. D 77, 064028 (2008), Conformally invariant massless spin-2 field in the de Sitter universe, arXiv:0805.2227.

[10] M.V. Takook, (2016), Quantum Field Theory in de Sitter Universe: ambient Space Formalism, arXiv:1403.1204.

[11] J. Bros, J.P. Gazeau, U. Moschella, Phys. Rev. Lett. 73, 1746 (1994), Quantum field theory in the de Sitter universe.

[12] J. Bros, U. Moschella, Fourier analysis and holomorphic decomposition on the one-sheeted hyperboloid, arXiv:math-ph/0311052v1].

[13] J. Bros, U. Moschella, Rev. Math. Phys. 8, 327 (1996), Two-point functions and quantum fields in de Sitter universe, gr-qc/9511019.

[14] F. Jalilifard, Y. Ahmadi, M.V. Takook, (2017), Electron-photon interaction in dS ambient space formalism, appears in MPLA.

[15] S. Helgason, Academi Press New York and London (1962), Differential Geometry and Symmetric Spaces.

[16] S. Helgason, American Mathematical Society Providence, Rhode Island (1994), Geometric Analysis on Symmetric Spaces.

[17] P. Bartesaghi, J.P. Gazeau, U. Moschella, M.V. Takook, Class. Quant. Grav. 18, 4373 (2001), Dirac fields and thermal effects in de Sitter universe.

[18] J.P. Gazeau, J. Renaud, M.V. Takook, Class. Quant. Grav. 17, 1415 (2000), Gupta-Bleuler quantization for minimally coupled scalar fields in de Sitter space, gr-qc/9904023]. 
[19] S. Rouhani, M.V. Takook, Quantum linear gravity in de Sitter universe On Bunch-Davies vacuum state, arXiv:1502.04814.

[20] N.A. Chernikov, E.A. Tagirov, Ann. Inst. H. Poincaré Sect. A 9, 109 (1968), Quantum theory of scalar fields in de Sitter space-time.

[21] M.V. Takook, Quantum de Sitter-black hole in ambient space formalism, Lecture presented at 5th Workshop and Seminar on Topics in Theoretical Physics, Azarbaijan Shahid Madani University, 23-25 August (2017), arXiv:1711.01067.

[22] F. Mandl, G. Shaw, John Wiley Sons, (2010), Quantum field theory.

[23] M. Kaku, Oxford university press, (1993), Quantum field theory A modern introduction.

[24] C. Itzykson, J.B. Zuber, McGRAW-HILL international book company, (1980), Quantum field theory. 